\title{
Activity-Dependent Development of Callosal Projections in the Somatosensory Cortex
}

\author{
Chun-Lei Wang, ${ }^{\star}$ Lei Zhang, ${ }^{*}$ Yang Zhou, Jing Zhou, Xiu-Juan Yang, Shu-min Duan, Zhi-Qi Xiong, and Yu-Qiang Ding \\ Institute of Neuroscience and Key Laboratory of Neurobiology, Shanghai Institutes for Biological Sciences, Chinese Academy of Sciences, Shanghai 200031, \\ China
}

The corpus callosum is the largest commissural system in the mammalian brain, but the mechanisms underlying its development are not well understood. Here we report that neuronal activity is necessary for the normal development and maintenance of callosal projections in the mouse somatosensory cortex. We labeled a subpopulation of layer II/III callosal neurons via in utero electroporation and traced their axons in the contralateral cortex at different postnatal stages. Callosal axons displayed region-and layer-specific projection patterns within the first 2 weeks postnatally. Prenatal suppression of neuronal excitation was achieved via electroporation-induced overexpression of the inward rectifying potassium channel Kir2.1 in layer II/III cortical neurons. This resulted in abnormal callosal projections with many axons extending beyond layers II-III to terminate in layer I. Others failed to terminate at the border between the primary and secondary somatosensory cortices. Blocking synaptic transmission via expression of the tetanus toxin light chain (TeNT-LC) in these axons produced a more pronounced reduction in the projections to the border region, and the eventual disappearance of callosal projections over the entire somatosensory cortex. When Kir2.1 and TeNT-LC were coexpressed, callosal axon targeting exhibited a more severe phenotype that appeared to represent the addition of the effects produced by individual expression of Kir2.1 and TeNT-LC. These results underscore the importance of activity in regulating the developing neural connections and suggest that neuronal and synaptic activities are involved in regulating different aspects of the development of callosal projection.

Key words: corpus callosum; development; neuronal activity; somatosensory cortex; barrel cortex; synaptic transmission; Kir 2.1

\section{Introduction}

The mammalian somatosensory cortex, including the primary (S1) and secondary (S2) cortices, is the cortical region that first receives and processes somatosensory information from the thalamus. The generation of cortical outputs in response to sensory inputs requires functional connections between the cerebral hemispheres. Such interhemispheric connections occur primarily via the axons of the corpus callosum. The adult S1 contains some callosally projecting neurons in layers II-III (Ivy et al., 1984; Hedin-Pereira et al., 1999; Brown and Dyck, 2005). During early postnatal development in rodents (Ivy and Killackey, 1981, 1982; O'Leary et al., 1981), however, layer II/III cortical neurons initially send exuberant callosal projections to the contralateral S1 that are later pruned to the adult projection pattern. In contrast to S1, the S1/S2 border region receives dense callosal projections in the early postnatal period that are retained through adulthood (Wise and Jones, 1976; Akers and Killackey, 1978; Ivy and Kil-

Received April 11, 2007; revised Aug. 28, 2007; accepted Aug. 31, 2007.

This work was supported by grants from the National Natural Science Foundation of China (30721004, 30525014, 30628016), "973" program and Key State Research Program from the Ministry of Science and Technology of China (2006CB806600, 2006CB943900), "Pujing Project" of Shanghai (06PJ14116), and the Chinese Academy of Sciences (12030303030KSCX1-YW-R). We thank Lily Jan and Heiner Niemann for reagents and Mark Jacquin, Linda Richards, and Mu-ming Poo for valuable comments on this manuscript.

${ }^{*}$ C.-L.W. and L.Z. contributed equally to this work.

Correspondence should be addressed to Dr. Yu-Qiang Ding, Institute of Neuroscience, Chinese Academy of Sciences, Shanghai 200031, China. E-mail: dingyq@ion.ac.cn.

DOI:10.1523/JNEUROSCI.3380-07.2007

Copyright $\odot 2007$ Society for Neuroscience 0270-6474/07/2711334-09\$15.00/0 lackey, 1981, 1982; O’Leary et al., 1981; Olavarria and Van Sluyters, 1995). Callosal connections in the somatosensory cortex are essential for the bilateral integration of cortical information (Shuler et al., 2001; Hlushchuk and Hari, 2006), and delineating the precise mechanisms underlying their development are important for understanding how complex long-range circuits are formed in the brain.

Previous studies have shown that neuronal activity is necessary for the normal development of axonal projections in the vertebrate visual system (Sur and Leamey, 2001; Hensch, 2005). Spontaneous neuronal activity before eye opening sculpts the initial connections, whereas evoked neuronal activity associated with the visual experience is vital for the refinement and maturation of these connections (Katz and Shatz, 1996). Blockade of NMDA receptors abolishes the pruning of retinal axon branches in the developing optic tectum of Xenopus tadpoles (Ruthazer et al., 2003). Suppression of neuronal activity in individual retinal ganglion cells inhibits their net growth and formation of new branches through an activity-based competitive mechanism (Hua et al., 2005). Neuronal activity is also involved in the pathfinding of developing spinal motor neuron axons in chick embryos (Hanson and Landmesser, 2004; Li et al., 2005), as well as in the establishment of olfactory sensory maps in mice (Yu et al., 2004). Thus, both neuronal and synaptic activities are important for the development of neuronal connections in developing sensory systems.

In the developing cerebral cortex, intrinsic spontaneous activ- 
ity in layer $\mathrm{V}$ pyramidal neurons is necessary for the formation of their ipsilateral and layer-specific axon arborizations in the somatosensory cortex (Dantzker and Callaway, 1998; Butler et al., 2001) and callosal projections in the visual cortex (Mizuno et al., 2007). Here we examined the role of neuronal and synaptic activities in the development of callosal connections in the somatosensory cortex, using in utero electroporation of exogenous constructs that perturb these activities in developing layer II/III cortical neurons. Our findings indicate that both electrical excitation and the synaptic outputs of these neurons are necessary for the development of their region- and layer-specific callosal projections in the somatosensory cortex.

\section{Materials and Methods}

Plasmid preparation. Expression plasmids encoding Kir2.1 (Kubo et al., 1993; Johns et al., 1999) or tetanus toxin light chain (TeNT-LC) (Hua et al., 1998; Burrone et al., 2002) fused with the Flag sequence (MDYKDDDDK) at the $\mathrm{N}$ terminal under the control of the CAG promoter were generated. A non-conducting form of Kir2.1 (mutant Kir2.1) was also generated in which three amino acids at the pore region were mutated (GYG to AAA) (Burrone et al., 2002). CAG-enhanced green fluorescent protein (EGFP) (Ding et al., 2004) was used to examine the normal development of callosal projections in the somatosensory cortex and to examine the consequences of expression of CAG-Kir2.1 and/or CAGTeNT-LC after developing callosal projections.

In utero electroporation. After anesthesia with sodium pentobarbital, pregnant mice at $15.5 \mathrm{~d}$ postcoitum (dpc) were subjected to abdominal incision to expose the uterus. Through the uterine wall, embryos were visualized, and plasmids ( $1.5 \mu \mathrm{g} / \mu \mathrm{l}$ for each) were injected into the lateral ventricle through a glass capillary. Electric pulses were then delivered to embryos by gently clasping their heads with forceps-shaped electrodes connected to a square-pulse generator, ECM-830 (BTX, Holliston, MA). Five $36 \mathrm{~V}$ pulses of $50 \mathrm{~ms}$ were applied at $1 \mathrm{~s}$ intervals. Uterine horns were repositioned into the abdominal cavity before the abdominal wall and the skin were sutured. Pups were reared to different postnatal stages. Under deep anesthesia, mice were perfused transcardially with saline followed by $4 \%$ paraformaldehyde in $0.1 \mathrm{~m}$ phosphate buffer ( $\mathrm{PB}), \mathrm{pH}$ 7.4. At each time point, at least six pups (three for control and three for expression of Kir2.1, TeNT-LC, or both, respectively) from two to four pregnant mice were used for data analysis in each set of experiments, and only the cases in which electroporated neurons occurred exclusively in the somatosensory cortex were included. Animal care and experimental protocols were approved by the Institute of Neuroscience, Chinese Academy of Sciences, China.

Immunostaining, in situ hybridization, and terminal deoxynucleotidyl transferase-mediated biotinylated UTP nick end labeling staining. Brains were cut into $20-\mu \mathrm{m}$-thick transverse sections with a cryostat after cryoprotection with $20 \%$ sucrose in PB. To visualize callosal axons in their entirety, immunostaining for EGFP was performed using standard methods. Briefly, brain sections were incubated overnight with rabbit anti-GFP antibody (1:2000; Invitrogen, Eugene, OR) at $4^{\circ} \mathrm{C}$, washed in $0.01 \mathrm{M}$ PBS, pH 7.4, incubated with secondary biotinylated goat antirabbit antibody (1:200; Vector Laboratories, Burlingame, CA) for $2 \mathrm{~h}$ at room temperature, washed again in PBS, and incubated with Cy2streptavidin (1:1000; Jackson ImmunoResearch, West Grove, PA) for 2 h. Sections were counterstained with Hoechst 33258 (Sigma, St. Louis, $\mathrm{MO})$ to clearly identify cortical layers and the morphological features defining S1 (see Results). Mouse anti-Flag antibody (1:200; Sigma) was used to examine whether all EGFP-expressing neurons contain Kir2.1 or TeNT-LC after cotransfection of EGFP with Kir2.1 or TeNT-LC. Sections were observed under confocal or eipifluorescent microscopy. Antisense RNA probes were used to reveal expression of Kir2.1 and TeNT-LC mRNA in the electroporated somatosensory cortex, and terminal deoxynucleotidyl transferase-mediated biotinylated UTP nick end labeling (TUNEL) staining was performed on some brain sections to assess possible electroporation-induced abnormal cell death, as described previously (Ding et al., 2003).

Slice preparation and electrophysiological recording. Brain slices from postnatal day 7 (P7) mice were prepared by the use of previously described methods (Chen et al., 2007). Kir2.1-overexpressing and control neurons in layers II-III were visualized via their green fluorescence, and whole-cell patch-clamp recordings were performed by the use of an Axopatch 700B amplifier (Molecular Devices, Sunnyvale, CA). Recording pipettes with resistances of 3-5 M $\Omega$ were pulled from borosilicate glass (P-97; Sutter Instruments, Novato, CA) and filled with a solution of (in mм) 130 potassium gluconate, $10 \mathrm{KCl}, 2 \mathrm{MgCl}_{2}, 0.2 \mathrm{EGTA}, 10 \mathrm{HEPES}, 2$ ATP, and 0.5 GTP. External artificial CSF (in mM: $119 \mathrm{NaCl}, 2.3 \mathrm{KCl}, 1.3$ $\mathrm{MgSO}_{4}, 2.5 \mathrm{CaCl}_{2}, 26.2 \mathrm{NaHCO}_{3}, 1 \mathrm{NaH}_{2} \mathrm{PO}_{4}$, and 11 glucose) was bubbled continuously with carbogen $\left(95 \% \mathrm{O}_{2} / 5 \% \mathrm{CO}_{2}\right)$. Resting membrane potential was measured after rupturing of cell membrane, and in most of the experiments, cells were held at $-70 \mathrm{mV}$. Data were analyzed with Clampfit 9.2 (Molecular Devices) and Igor 4.09 (WaveMetrics, Lake Oswego, OR); statistical results were presented as mean \pm SE; significant differences were revealed by two-tailed Student's $t$ tests (Graph Pad, San Diego, CA).

Morphological analyses. For analysis of callosal axon growth in the corpus callosum at $\mathrm{P} 4$, one brain section at the approximate level of bregma -1.58 (Paxinos and Franklin, 2001) was selected from each mouse brain electroporated with Kir2.1 and EGFP ( $n=3$ for each). Distances of three pioneer callosal axons from the midline were measured and averaged. A comparison with similarly selected control axons was performed with a Student's $t$ test. For analysis of callosal ingrowth into the contralateral somatosensory cortex at P7-P9, brain sections were selected in the same way from each brain electroporated with Kir2.1 $(n=$ $4)$, TeNT-LC $(n=3)$, and EGFP $(n=4)$, respectively, and axons in layer IV of $\mathrm{S} 1$ and in the S1/S2 border region were counted. Because callosal axons traverse layer IV before terminating in layers II-III, axon counts in layer IV provided a valid measure of changes in callosal axon invasion en masse (see Results). For the S1/S2 border region, axons were counted in $\sim 500$ - $\mu \mathrm{m}$-wide blocks centered on the lateral most $\mathrm{S} 1$; this area corresponds to the territory normally receiving a dense callosal projection, as revealed by EGFP expression (see Results). For S1, we counted axons in a region corresponding to the representation of the middle row/arc of mystacial whiskers; this S1 region at the approximate level of bregma -1.58 was $\sim 1500 \mu \mathrm{m}$ wide. The counts from the $S 1$ region were divided by 3 , and the numbers of axons per $500-\mu \mathrm{m}$-wide area were obtained. The numbers of axons in layer IV of S1 and the S1/S2 border regions (referred to as cortical invasion index) were statistically evaluated with a Student's $t$ test. For analysis of the accuracy of layer targeting by callosal projections, one section at the approximate level of bregma -1.58 was selected from each of the brains transfected, respectively, with Kir2.1, TeNT-LC, and EGFP. The S1 and S1/S2 border regions were equally divided into 20 bins across all of layers $\mathrm{I}-\mathrm{V}$, and Cy2 fluorescent intensity in each bin was measured and normalized by Image-Pro Plus 5.0. Normalized data were pooled and compared with those from controls by the use of a Student's $t$ test ( $n=4$ for each).

\section{Results \\ Normal development of region- and layer-specific callosal axon projection}

Although callosal connections have been described in the somatosensory cortex (Ivy et al., 1984; Hedin-Pereira et al., 1999; Brown and Dyck, 2005), their termination sites and development have not been fully characterized. We thus began our study by examining the development of axonal projections from layer II/ III pyramidal neurons that send the majority of callosal axons to the contralateral somatosensory cortex. The location of the somatosensory cortex was determined according to the criteria described by Paxinos and Franklin (2001), with S1 and S2 clearly distinguished by Hoechst couterstaining. As shown in Figures $1 C-J$ and $2 A-D, S 1$ is readily identified by its densely packed cells in layer IV before P5 and pronounced whisker-associated barrels thereafter, whereas S2 (located lateral to S1) displays neither of these properties. To label layer II/III pyramidal neurons of the somatosensory cortex, plasmids encoding EGFP were electropo- 
rated in utero into the dorsolateral ventricular zone of the mouse forebrain at 15.5 dpc (Hatanaka et al., 2006). After electroporation, a significant number of EGFPexpressing neurons migrated to the superficial region of the cortex by $\mathrm{P} 1$. Most labeled cells had completed their migration into layers II-III by P3 (Fig. $1 A, B$ ), and all were located in these layers after $\mathrm{P} 6$ (Fig. $2 C$; see Figs. $4 A, 5 A$ ).

Labeled callosal axons were first observed at P2 as they coursed medially and approached the midline (Fig. $1 A$ ). Callosal axons crossed the midline at P3 (Fig. $1 B$ ), coursed beneath the gray matter of the contralateral somatosensory cortex at P5 (Fig. 1C), and penetrated the gray matter at P6 (Fig. 1D). Callosal invasion of the gray matter was first seen in medial S1 and spread laterally to the S1/S2 border region with development. After P8, projection patterns in S1 and the S1/S2 border region exhibited marked differences: callosal axons rapidly and densely invaded the S1/S2 border region by $\mathrm{P} 9$, with termination sites in layers I-III and V (Fig. 1G,H), whereas their further invasion of S1 was gradual and termination sites were mainly restricted to layers II-III (Fig. 1G,H). These projection patterns appeared to reach maturity by P12 (Fig. 1I,J). Together, these studies showed the normal development of region- and layer-specific projections of the callosal system during the first 2 postnatal weeks, with a moderately dense projection to layers II-III of S1 and a more dense projection to layers I-III and V of the S1/S2 border region.

\section{Assays of Kir2.1 overexpression in the somatosensory cortex}

To examine whether neuronal electrical activity plays a role in callosal axon development in the somatosensory cortex, we overexpressed the Flag-tagged inward rectifying potassium channel Kir2.1 (Kubo et al., 1993; Johns et al., 1999) in layer II/III pyramidal neurons via in utero electroporation. In situ hybridization of electroporated cortex showed that Kir2.1 mRNA expression was located exclusively in layers II-III of the somatosensory cortex at P8, P12, P21, and P25 (see supplemental Fig. 1C, available at www.jneurosci.org as supplemental material, and data not shown). Neurons exhibiting EGFP fluorescence expressed functional Kir2.1, as determined by anti-Flag antibody and by wholecell patch clamping of EGFP-positive neurons in P7 cortical slices (see supplemental Fig. 1A,B, available at www.jneurosci.org as supplemental material). Overexpression of Kir2.1 hyperpolarized neurons by shifting the average resting membrane potential from -60 to $-81 \mathrm{mV}$ (see supplemental Fig. $1 B$, available at www.jneurosci.org as supplemental material). It also increased the threshold for evoking action potentials: a larger depolarizing current $(\sim 110 \mathrm{pA})$ was required to evoke an action potential compared with that for control neurons $(\sim 40 \mathrm{pA})$ (see supplemental Fig. $1 B$, available at www.jneurosci.org as supplemental material). This is consistent with previous reports that overexpression of Kir2.1 in neurons reduces their spontaneous and evoked activity (Yu et al., 2004; Cancedda et al., 2007; Mizuno et al., 2007). Together, Kir2.1 overexpression specifically in layer II/III cortical neurons effectively suppressed their spontaneous and evoked electrical activity in vivo.

\section{Overexpression of Kir2.1 impedes callosal axon growth}

Cortical neurons cotransfected with Kir2.1 and EGFP migrated to layers II-III with a time course similar to that of control neurons transfected with EGFP alone (Fig. $2 A-D$; see Fig. $4 A, B$ ), suggesting that impaired neuronal excitation did not affect the radial migration of cortical neurons. Initial outgrowth of callosal axons on the ipsilateral side to electroporation was indistinguishable from littermate control axons (data not shown). However, Kir2.1-expressing axons lagged behind the control axons in their midline crossing at P3 and P4 (Fig. 3A,B). In addition, fewer Kir2.1 axons were found in the gray matter of $\mathrm{S} 1$ at P7 and later compared with control axons (Fig. $3 C, D$; see Fig. $5 K$ ). Similarly, 

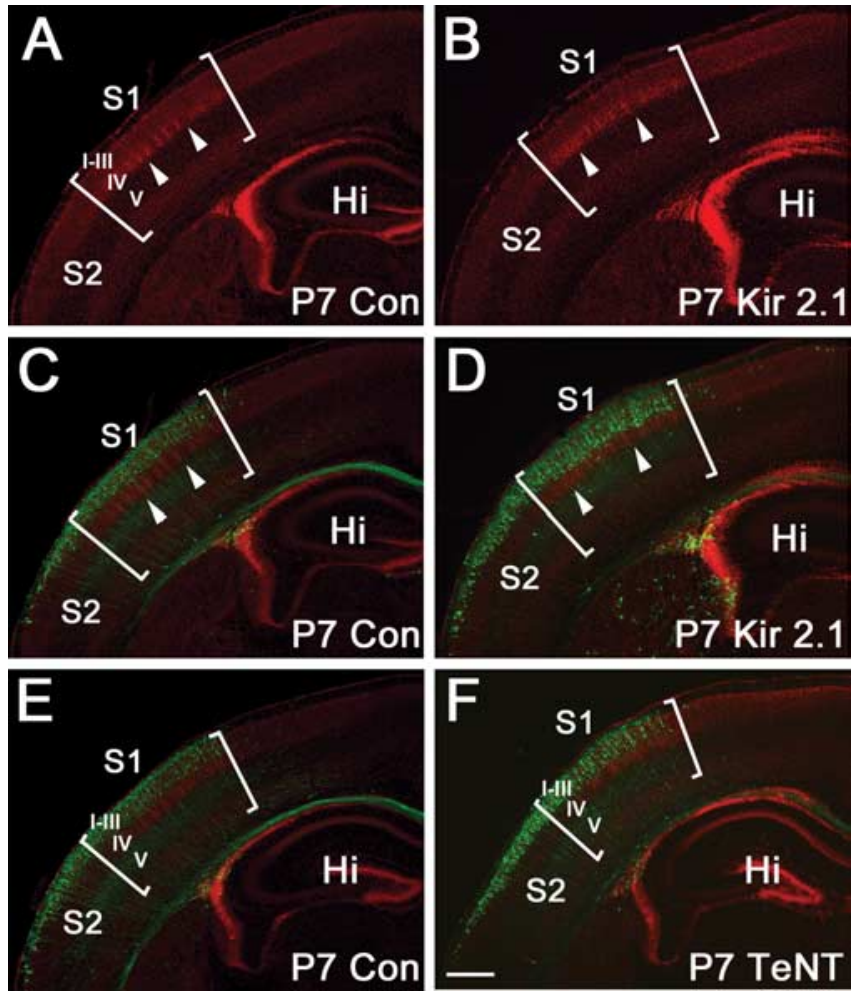

Figure 2. Expression of Kir2.1 or TeNT-LC did not affect the radial migration of cortical neurons in the somatosensory cortex. $\boldsymbol{A}-\boldsymbol{D}$, Kir2.1-expressing $(\boldsymbol{B}, \boldsymbol{D})$ and EGFP-expressing $(\boldsymbol{A}, \boldsymbol{C})$ neurons were located in layers II-III of S1 and S2 at P7. Sections were counterstained with Hoechst (red), and the $S 1$ region was delineated by whisker-associated barrels (arrowheads). $\boldsymbol{A}$ and $\boldsymbol{C}$, and $\boldsymbol{B}$ and $\boldsymbol{D}$, are the same field, respectively. $\boldsymbol{E}, \boldsymbol{F}$, TeNT-LC-expressing $(\boldsymbol{F})$ and EGFPexpressing $(\boldsymbol{E})$ neurons were located in layers II-III of S1 and S2 at P7. Hi, Hippocampus; Con, control. Scale bar, $550 \mu \mathrm{m}$.

fewer Kir2.1 axons were observed in the S1/S2 border region than control axons (Fig. 3D; see Fig. 5L). Thus, Kir2.1 overexpression in callosal neurons induces a delay in midline crossing and a reduction in the numbers of callosal axons entering the gray matter of $\mathrm{S} 1$ and the S1/S2 border region.

\section{Kir2.1 overexpression disrupts layer- and region-specific} callosal axon projection

Callosal axons normally display region- and layer-specific targeting in the somatosensory cortex during the second postnatal week. Unlike control callosal axons, which first ramify in layers II-III of S1 at P8-P9, Kir2.1-expressing axons traversed these layers without obvious arborization and terminated in layer I (see Fig. $8 A, B)$. The number of Kir2.1 axons in S1 was comparable to that of control axons at P9 (see Fig. $5 K$ ), although, as noted above, the timing of such ingrowth was delayed (Fig. 3). By P10P12, Kir2.1 axons extensively arborized in layer I of S1, particularly in its most superficial portion where control axon branching was not observed (Fig. 4C-F). This aberrant axon arborization in the superficial layer I appeared to be accompanied by a reduced axon branching in layers II-III and V (Fig. $4 C-G$ ). At P25, the latest time point studied, the layer I-targeting preference persisted, although slightly more extensive axon arbors occurred in layers II-III (data not shown). Thus, suppression of electrical excitation in callosal axons caused defects in layer-specific targeting in S1.

In addition to the layer I-targeting preference in S1, Kir2.1expressing callosal axons also displayed targeting errors at the
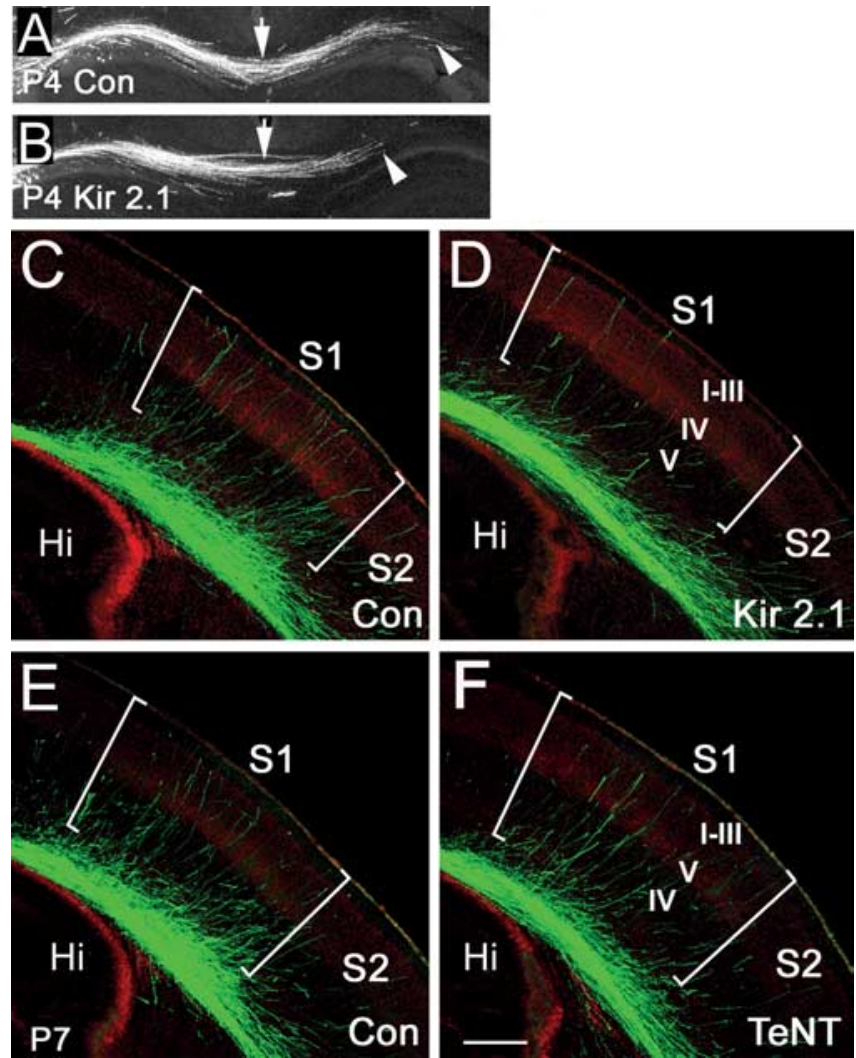

Figure 3. Overexpression of Kir2.1 delayed callosal axon elongation in the corpus callosum and expression of Kir2.1 or TeNT-LC slowed callosal invasion of the contralateral somatosensory cortex during the first postnatal week. $\boldsymbol{A}, \boldsymbol{B}$, Kir2.1-expressing callosal axons lagged behind control axons in the corpus callosum at P4 ( $\boldsymbol{A}, \boldsymbol{B}$, arrowheads). The distance of leading growth cones was measured from the midline and showed a significant difference between Kir2.1 and control axons ( ${ }^{*} p<0.001$ ). Arrows in $\boldsymbol{A}$ and $\boldsymbol{B}$ point to the midline. C, D, Callosal invasion of the gray matter in the contralateral cortex was slowed by overexpression of Kir2.1 at P7 (D) compared with that of control axons ( $\boldsymbol{C}$. $\boldsymbol{E}, \boldsymbol{F}$, Callosal invasion of the gray matter in the contralateral cortex was reduced by misexpression of TeNT-LC at P7 (F) compared with that of control axons (E). Con, Control; Hi, hippocampus. Scale bar, $350 \mu \mathrm{m}$.

S1/S2 border. Whereas callosal axons normally project promptly and extensively at the S1/S2 border by P8-P9 (Figs. $1 F, G, 5 L$; see Fig. $8 A$ ), Kir2.1 axons showed an unusually sparse projection to this area (Fig. $5 \mathrm{~L}$; see Fig. $8 \mathrm{~B}$ ), a situation found to persist at P12 and P25 (Fig. 4D) (data not shown). As was the case in S1, the majority of Kir2.1 axons bypassed layers II-III and terminated densely in layer I of the S1/S2 border region (Fig. $4 D, I, J$ ). These findings indicate that suppression of electrical excitation in callosal axons produces region- and layer-specific targeting errors.

To test the possibility that the observed defects in callosal axon development were caused by other confounding effects of Kir2.1 overexpression unrelated to its channel function, a plasmid encoding a mutated form of Kir2.1 lacking the channel activity was electroporated in otherwise normal and age-matched littermates in utero. Consistent with that observed in the mouse visual cortex (Mizuno et al., 2007), we found that overexpression of the mutant Kir2.1 did not affect the radial migration of transfected cortical neurons or the growth or pathfinding of their axons (data not shown).

Blocking transmitter release leads to different defects in callosal axon projection

The effects of Kir2.1 overexpression described above could be attributed to the suppressed neuronal excitation or to the failure of establishing proper synaptic transmission as a result of reduced 

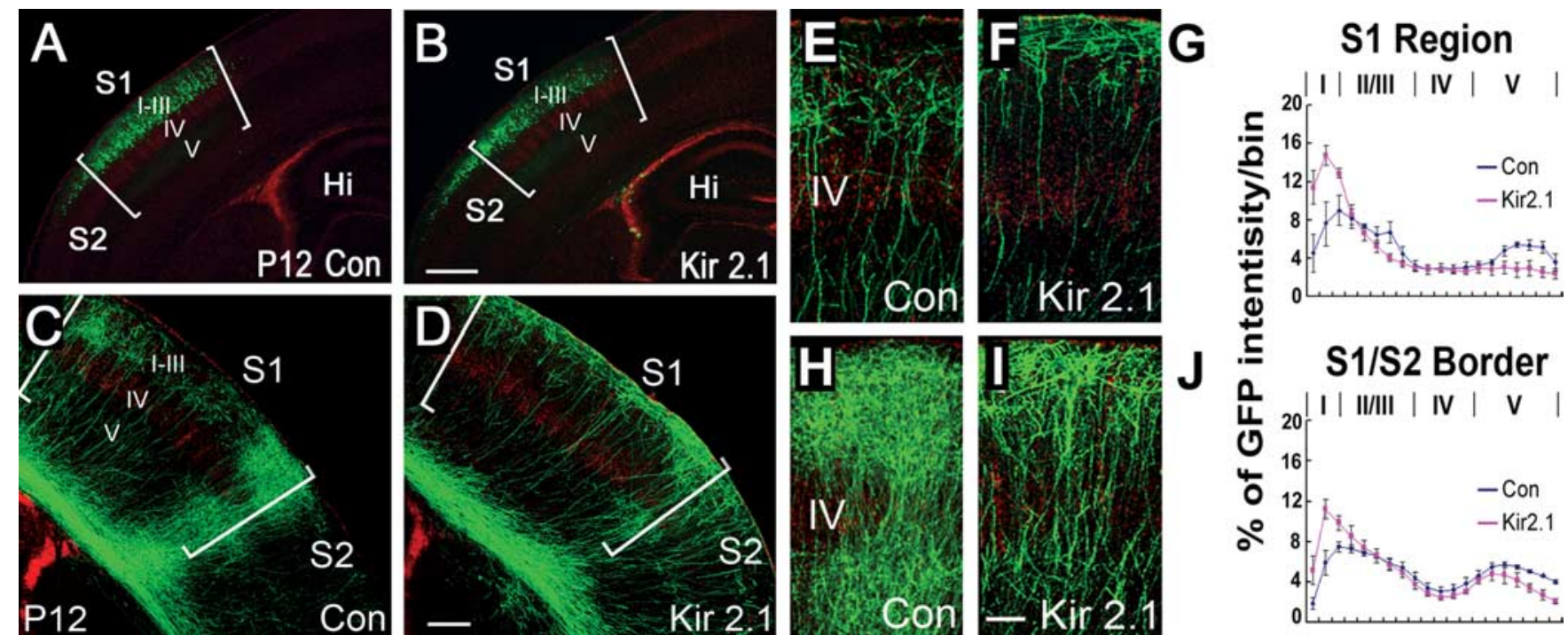

Figure 4. Overexpression of Kir2.1 led to the disruption of layer- and region-specific callosal projections in the contralateral somatosensory cortex at P12.A, B, Cortical neurons transfected with EGFP $(\boldsymbol{A})$ and Kir2.1 (B) were located in layers II-III of S1 and S2. $\boldsymbol{C}-\boldsymbol{J}$, At P12, control callosal axons terminated primarily in layers II-III of $S 1(\boldsymbol{C}, \boldsymbol{E})$, whereas Kir2.1-expressing axons bypassed these layers and terminated in layer I $(\boldsymbol{D}, \boldsymbol{F})$. At the $\mathbf{S 1} / \mathbf{S} 2$ border, Kir2.1-expressing axons $(\boldsymbol{D})$ were lower than controls $(\boldsymbol{C})$, and they tended to terminate preferentially in layer I as well $(\boldsymbol{I})$. $\boldsymbol{E}$, $\boldsymbol{F}$, High magnification of control ( $\boldsymbol{C}$ ) and Kir2.1-expressing (D) axons in S1. $\boldsymbol{H}, \boldsymbol{I}$, High magnification of control $(\boldsymbol{C})$ and Kir2.1-expressing (D) axons at the S1/S2 border. $\mathbf{G}, \boldsymbol{J}$, Layer distribution of control and Kir2.1-expressing axons in $S 1$ and at the $S 1 / S 2$ border. Normalized intensities of immunofluorescence represent densities of callosal axons in different layers of the somatosensory cortex. Con, Control; Hi, hippocampus. Scale bars: $A, B, 550 \mu \mathrm{m} ; C, D, 300 \mu \mathrm{m} ; E, F, H, I, 100 \mu \mathrm{m}$.
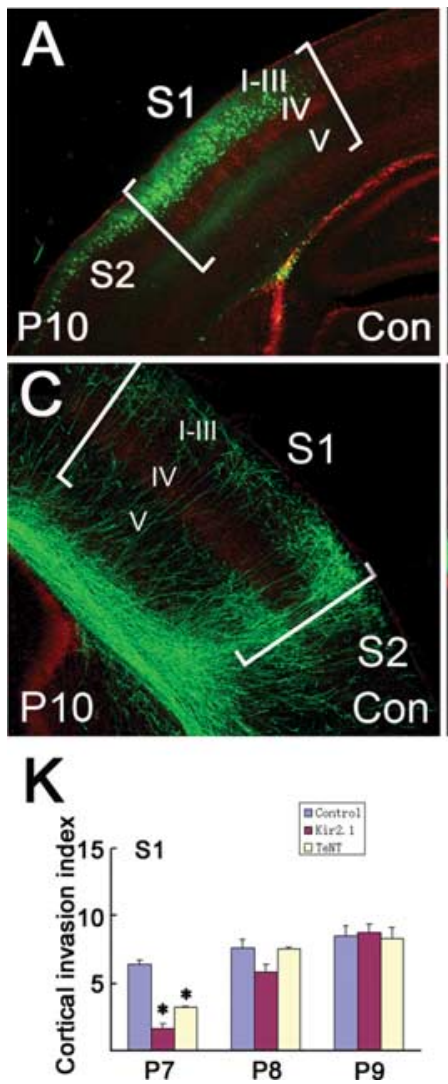
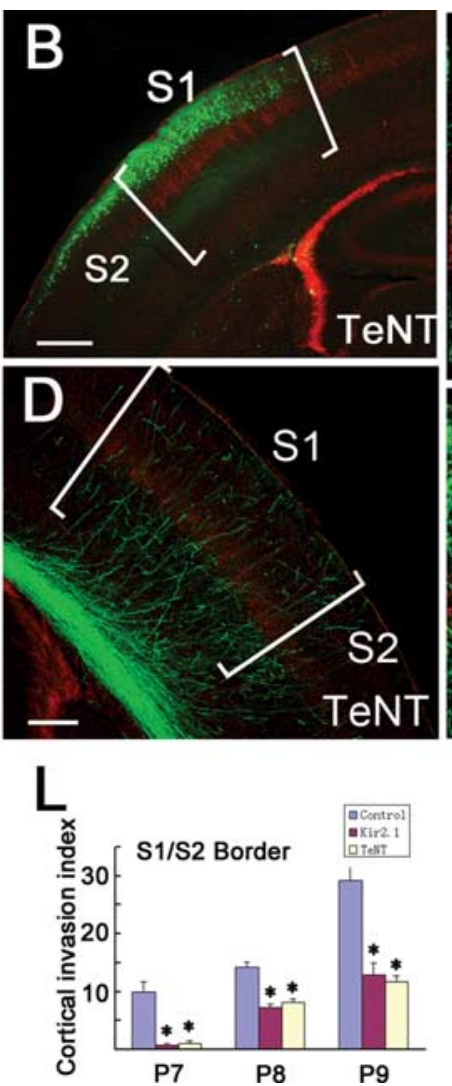
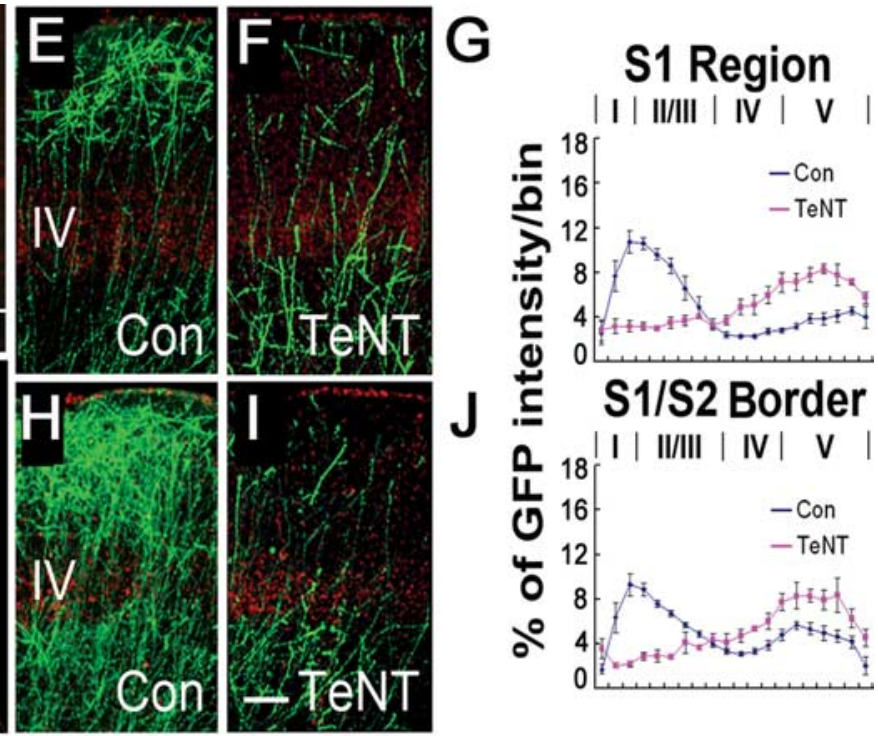

Figure 5. Expressing TeNT-LC resulted in aberrant callosal projections in the somatosensory cortex at P10. $\boldsymbol{A}, \boldsymbol{B}$, Cortical neurons transfected with EGFP $(\boldsymbol{A})$ and TeNT-LC $(\boldsymbol{B})$ were located in layers II-III of S1 and S2 at P10. $\boldsymbol{C}-\boldsymbol{J}$, In S1, control callosal axons primarily terminated in layers II-III $(\boldsymbol{C}, \boldsymbol{E})$, whereas TeNT-LC-expressing axons exhibited poor arborization in these layers $(\boldsymbol{D}, \boldsymbol{F})$. At the $S 1 / \boldsymbol{S} 2$ border, dense callosal projections were found in control $(\boldsymbol{C}, \boldsymbol{H})$ but not in TeNT-LC $(\boldsymbol{D}, \boldsymbol{I})$ expression. $\mathbf{G}, \boldsymbol{J}$, Layer distributions of control and TeNT-LC-expressing callosal axons in S1 and the S1/S2 border region were shown by normalized immunofluorescence intensities of callosal axons. $\boldsymbol{K}$, In S1, after expression of Kir 2.1 and TeNT-LC in layer II/III cortical neurons, their callosal invasion of the contralateral cortex was decreased at P7 ( $\left.{ }^{*} p<0.01\right)$, but it reached a similar level as control axons at P8 and P9. L, Expression of Kir 2.1 and TeNT-LC dramatically reduced callosal projections to the $S 1 / S 2$ border region at $P 7-P 9\left(^{*} p<0.01\right)$. The cortical invasion index was determined by the numbers of collosal axons per a 500 - $\mu \mathrm{m}$-wide region of layer IV of $S 1$ and the $S 1 / S 2$ border region (see Materials and Methods). Scale bars: $\boldsymbol{A}, \boldsymbol{B}, 450 \mu \mathrm{m} ; \boldsymbol{C}, \boldsymbol{D}, 250 \mu \mathrm{m} ; \boldsymbol{E}, \boldsymbol{F}, \boldsymbol{H}, \boldsymbol{I}, 120 \mu \mathrm{m}$. 
firing of callosal axons. To distinguish between these two possibilities, we specifically blocked the transmitter release from developing cortical neurons by in utero electroporation of the TeNT-LC, which cleaves the vesicle-associated membrane protein complex that is necessary for normal vesicular exocytosis (Hua et al., 1998; Yu et al., 2004; Hua et al., 2005). Immunostaining and in situ hybridization assays indicated that TeNT-LC was expressed in layers II-III of the somatosensory cortex after in utero electroporation (see supplemental Fig. $2 A-C$, available at www.jneurosci.org as supplemental material). Expression of TeNT-LC did not affect the radial migration of the cortical neurons (Fig. 2 F; see also supplemental Fig. $2 D-F$, available at www. jneurosci.org as supplemental material), nor their initial axon outgrowth, midline crossing, and penetration of the contralateral white matter at P2-P5 (data not shown). However, we found that at $\mathrm{P} 6-\mathrm{P} 7$ the invasion of the gray matter in both the S1 and S1/S2 border regions was delayed (Figs. $3 F, 5 K, L$ ). Thus, transmitter release is not required for callosal axon elongation but is necessary for the timely projection to their targets.

Expression of TeNT-LC had more profound effects during the second postnatal week. In S1, although a large number of TeNTLC-expressing axons projected to contralateral layers II-III, they failed to ramify there. The conspicuous absence of terminal arborization (Fig. 5D,F; see Fig. 8C,F) markedly differs from the Kir2.1 phenotype, where callosal axons showed extensive arborization in layer I after bypassing layers II-III (Fig. 4). At the S1/S2 border, only a few TeNT-LC-expressing callosal axons were observed (Fig. 5D, I, L; see Fig. $8 C$, $J$ ), and the reduction in axon number is more severe than that observed for Kir2.1expressing axons (Fig. 4). Moreover, the numbers of TeNT-LCexpressing callosal axons in the somatosensory cortex decreased significantly after P10 and were nearly absent by P21 (Fig. $6 B, D, F)$. This gradual withdrawal of callosal axons from the entire somatosensory cortex cannot be simply explained by abnormal cell death caused by TeNT-LC transfection, because a significant number of TeNT-LC-expressing neurons remained in layers II-III of the electroporated side (Fig. 6A, C,E; see also supplemental Fig. 2, available at www.jneurosci.org as supplemental material) and no obvious increase in TUNEL-positive neurons in TeNT-LC-electroporated cortex was found compared with control cortex (data not shown). Thus, blocking transmitter release produces a paucity of callosal arbors and projections to the contralateral cortex and an eventual loss of these projections during early postnatal development. The latter result suggests that proper synaptic activity is required for the maintenance of callosal projections in the somatosensory cortex.

\section{Coexpression of Kir2.1 and TeNT-LC results in additive effects on callosal axon projection}

Development of callosal projections was also examined for layer II/III cortical neurons transfected with both Kir2.1 and TeNTLC. Cortical neurons expressing both proteins migrated normally to layers II-III (Fig. 7D). Initially, their callosal projections resembled those expressing Kir2.1 alone, with delayed midline crossing and ingrowth of callosal axons into the contralateral gray matter through P7 (data not shown). However, additive effects of Kir2.1/TeNT-LC coexpression were observed at later stages of development. The number of callosal axons was more drastically reduced in both $\mathrm{S} 1$ and the S1/S2 border region at P9 compared with that found for axons transfected with each protein alone (Fig. $8 A-D$ ). Yet, the few remaining axons found in the contralateral S1 bypassed layers II-III and arborized in layer I (Fig. $8 D, H$ ), resembling the Kir2.1 (Fig. $8 B, F$ ) rather than the TeNT-LC (Fig.
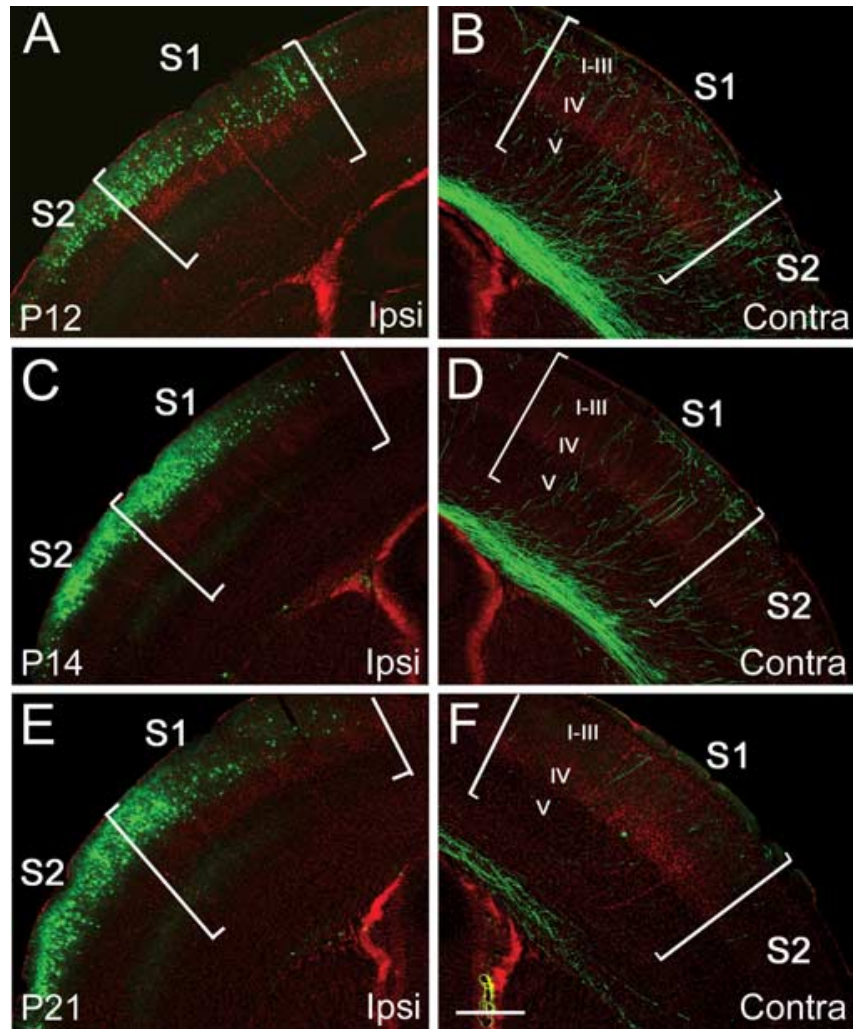

Figure 6. Expression of TeNT-LC caused a failure of maintenance of callosal axons in the contralateral somatosensory cortex. $\boldsymbol{A}-\boldsymbol{F}$, After expression of TeNT-LC in layer II/III cortical neurons $(\boldsymbol{A}, \boldsymbol{C}, \boldsymbol{E})$, their axons in the contralateral cortex were greatly reduced at $\mathrm{P} 12(\boldsymbol{B})$ and $\mathrm{P} 14$ (D) and almost completely lost at P21 $(\boldsymbol{F})$. For comparison with normal callosal projections, see Figure 1. Ipsi, Ipsilateral side to electroporation; Contra, contralateral side to electroporation. Scale bar, $500 \mu \mathrm{m}$.
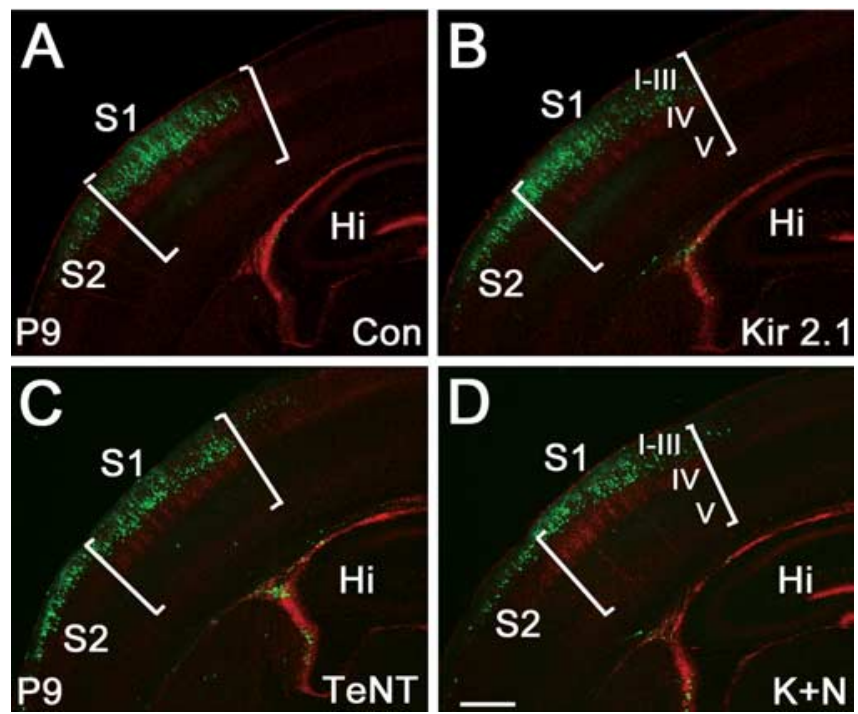

Figure 7. Coexpression of Kir2.1 and TeNT-LC in layer II/III neurons of the somatosensory cortex at P9. Layer distribution of these cotransfected neurons $(K+N ; D)$ was similar to that of cortical neurons transfected with $\operatorname{EGFP}(\boldsymbol{A})$, Kir2.1 (B), and TeNT-LC $(\boldsymbol{C})$ alone in the somatosensory cortex. Hi, Hippocampus; Con, control. Scale bar, $650 \mu \mathrm{m}$.

$8 C, G)$ phenotype. The S1/S2 border region displayed a paucity of callosal axon invasion (Fig. 5D,L), similar to the single transfection phenotype (Fig. 5D,J,K). Subsequently, callosal axons were further diminished in density by $\mathrm{P} 10$ and almost completely eliminated by 

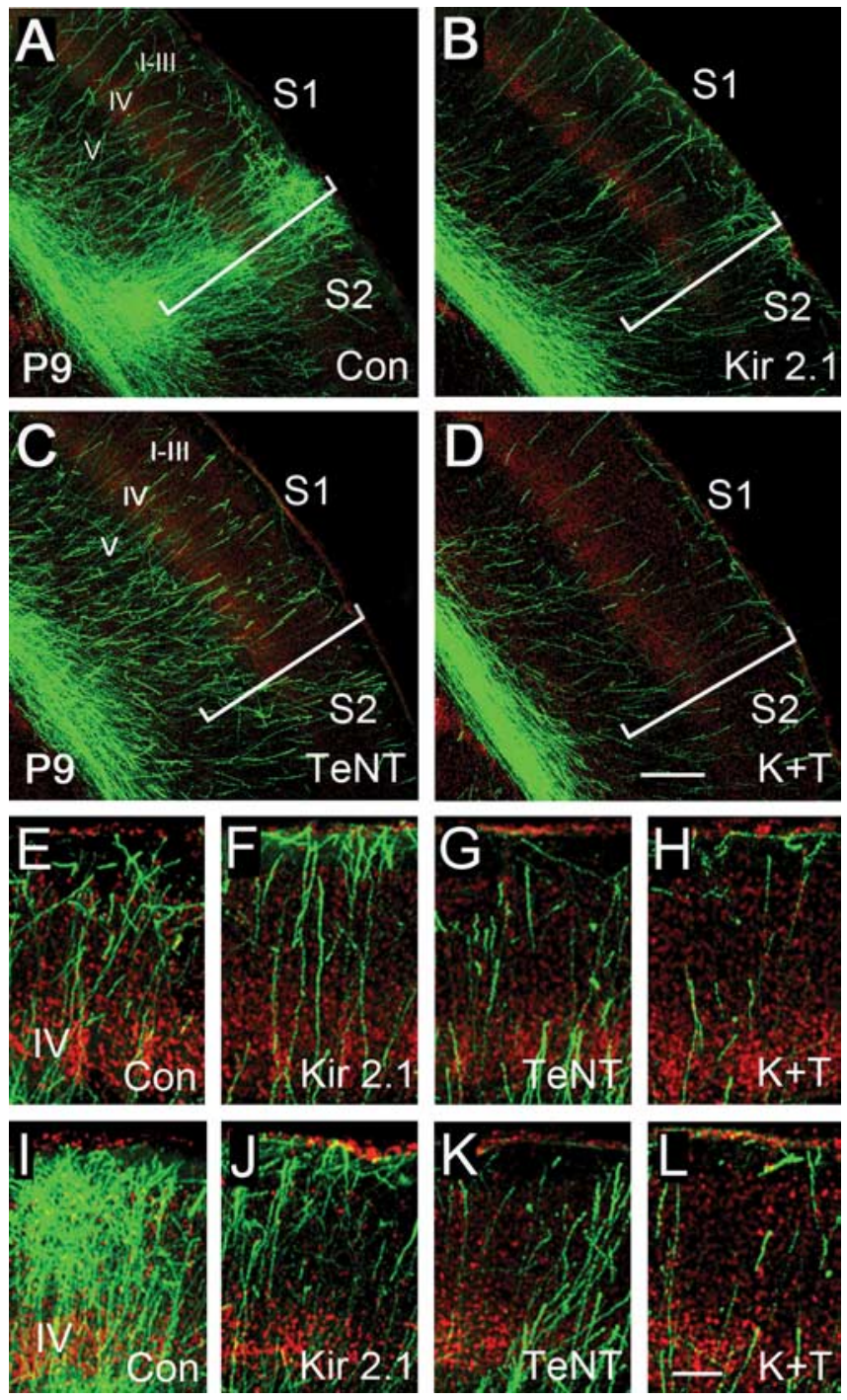

Figure 8. Coexpression of Kir2.1 and TeNT-LC in callosal neurons caused more severe defects in the development of callosal projections in the somatosensory cortex than those of individual transfections at P9. $\boldsymbol{A}-\boldsymbol{D}$, Kir2.1/TeNT-LC-coexpressing callosal axons were more dramatically reduced in both $\mathrm{S1}$ and the $\mathrm{S1/S2}$ border region $(\boldsymbol{D})$ compared with control $(\boldsymbol{A})$, Kir2.1 $(\boldsymbol{B})$, and TeNT-LC $(\boldsymbol{C})$ axons. It should be noted that these reduced callosal axons in $\mathrm{S} 1$ bypassed layers $\|-I I l$ and ended in layer I $(\boldsymbol{D}, \boldsymbol{H})$. The $S 1$ regions in $\boldsymbol{A}-\boldsymbol{D}$ were enlarged in $\boldsymbol{E}-\boldsymbol{H}$, respectively. The $\mathbf{S 1 / S 2}$ border regions in $\boldsymbol{A}-\boldsymbol{D}$ were enlarged in $\boldsymbol{I}-\boldsymbol{L}$, respectively. Scale bars: $\boldsymbol{A}-\boldsymbol{D}, 350 \mu \mathrm{m} ; \boldsymbol{E}-\boldsymbol{L}$, $100 \mu \mathrm{m}$. Con, Control; K+T, Kir2.1 plus TeNT-LC.

P14 (data not shown). Thus, simultaneous suppression of electrical excitation and synaptic transmission produced a more profound reduction in callosal projections in the somatosensory cortex than either manipulation alone. The observation of callosal axons bypassing layers II-III and arborizing in layer I in single Kir2.1 expression and coexpression of both proteins suggests that this overshooting phenotype results specifically from reduced neuronal activity, rather than impaired transmitter release.

\section{Discussion}

The use of in utero electroporation of EGFP in callosal neurons of the somatosensory cortex allowed our study on the role of neuronal and synaptic activities in the development of callosal projections. Within the first 2 postnatal weeks, contralaterally projecting layer II/III neurons have established their projections with a moderate density in layers II-III of S1 and a high density in layers I-III and V of the S1/S2 border region. Suppression of neuronal excitation via Kir2.1 overexpression resulted in a significant reduction in callosal projections to the S1/S2 border region and overshooting projections to layer I of both S1 and the S1/S2 border. Similarly, blockade of transmitter release from these callosal axons via expression of TeNT-LC eliminated the initial dense callosal projections to the S1/S2 border region and resulted in poor axon arborization in both $\mathrm{S} 1$ and S1/S2 border and an eventual complete elimination of these projections over the entire somatosensory cortex. The failure to maintain callosal axons led to the development of a somatosensory cortex mostly devoid of callosal inputs. The phenotypes caused by expression of Kir2.1, TeNT-LC, and both of them are summarized in Figure 9. On the basis of these findings, we concluded that neuronal activity and transmitter release, either spontaneously or evoked by action potentials, are necessary for the initial development and maintenance of callosal projections to the somatosensory cortex.

\section{Development of callosal projections to somatosensory and visual cortices}

The barrel cortex (S1) has long been considered acallosal, but more recent studies suggest that callosal neurons do project to S1 (Ivy et al., 1984; Hedin-Pereira et al., 1999; Brown and Dyck, 2005). Retrograde tracing studies have found that callosal neurons are numerous in layers II-III of the S1/S2 border region in rat and some are present in mouse S1 (Ivy and Killackey, 1981; Brown and Dyck, 2005). An anterograde study in hamster shows that callosal neurons in the parietal cortex terminate in layers II-III and V of the contralateral cortex (Hedin-Pereira et al., 1999). Our findings on layer- and region-specific projections of callosal axons during postnatal development are consistent with these previous observations on the somatosensory cortex and a recent report on the development of callosal projections in the mouse visual cortex (Mizuno et al., 2007).

In normal development, the formation of callosal projections in the somatosensory cortex exhibits a similarity to that found in the visual cortex (Mizuno et al., 2007): axons cross the midline at P3 and display extensive terminal arborization in the contralateral cortex during the second postnatal week (P7-P13). However, there are some differences between the two sensory cortices. In the visual cortex, there is a 2-3 d "waiting period" (P5-P7) between the arrival of callosal axons and the onset of their invasion into the gray matter (Mizuno et al., 2007). In the somatosensory cortex, no such waiting period was observed (the arrival of callosal axons at P5 is followed by the invasion of the gray matter at P6) (Fig. 1). In addition, callosal projections in the visual cortex are only found in the border region between area 17 (primary visual cortex) and area 18 (secondary visual cortex) (Innocenti and Price, 2005; Mizuno et al., 2007), whereas they are present in both $\mathrm{S} 1$ and the S1/S2 border region. These differences may reflect distinct functional requirements for the integration of somatosensory versus visual signals of two hemispheres and suggest the existence of cortical area-specific mechanisms for directing the formation of callosal connections.

Requirement of electrical activity for callosal axon projection In vitro evidence has shown that intrinsic spontaneous neuronal activity is required for cortical layer VI neurons to develop their ipsilateral layer-specific arbors (Butler et al., 2001) and for layer II/III neurons to extend their ipsilateral axonal branches (Uesaka et al., 2005, 2006). Altering primary afferent inputs to the somatosensory cortex by early sectioning of the infraorbital nerve, which conveys sensory information from the mystacial vibrissas, 

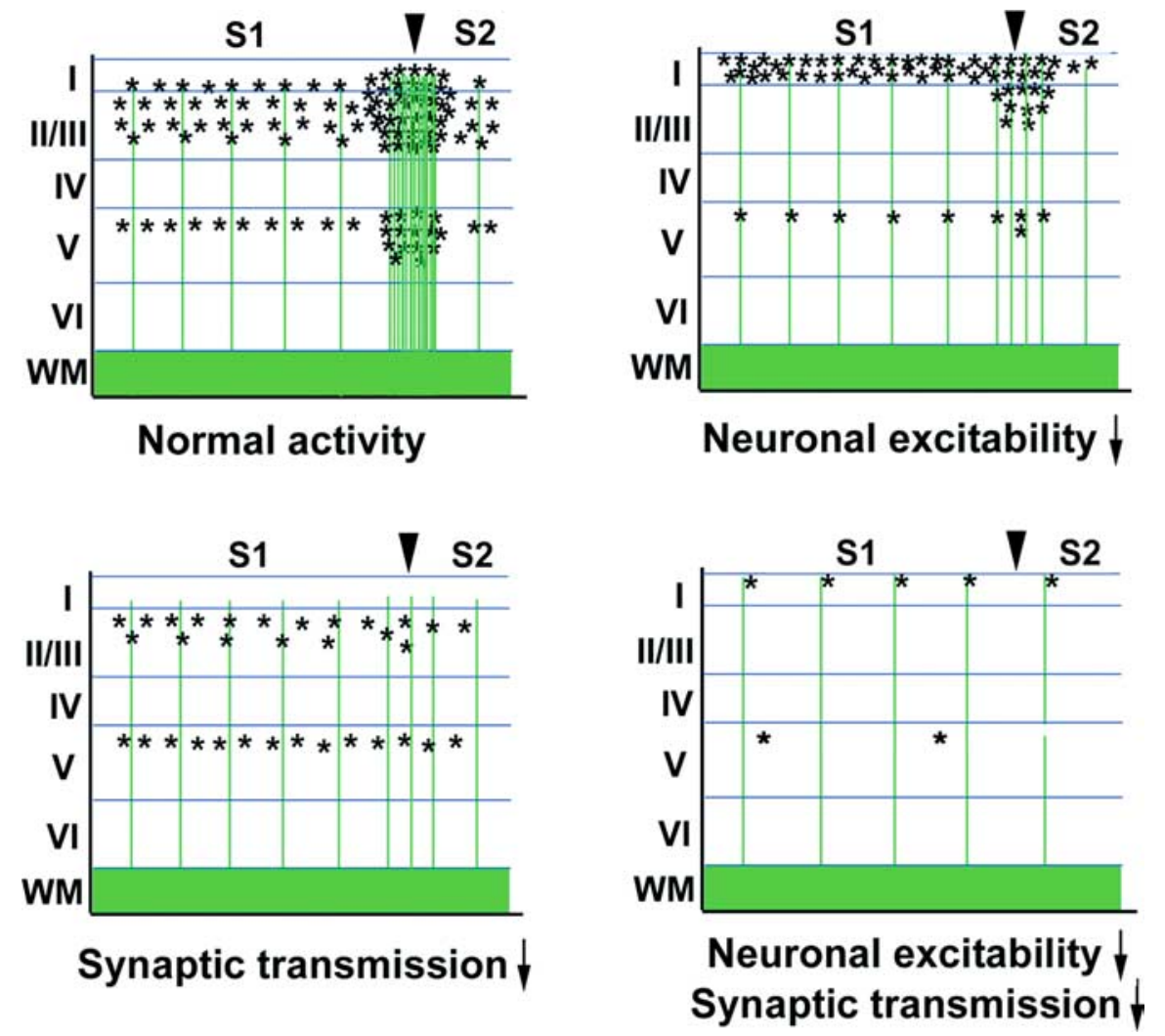

Figure 9. A summary of callosal projection phenotypes after expression of Kir2.1, TeNT-LC, and both of them in layer II/II pyramidal neurons of the somatosensory cortex. Reducing neuronal excitability and blocking synaptic transmission leads to a great reduction in callosal projections to the $\$ 1 / S 2$ border regions, and simultaneous manipulation results in a more pronounced reduction in the projections to the border region. Whereas reducing excitability in callosal neurons causes an overshooting phenotype in callosal layer targeting, blocking synaptic transmission impairs normal callosal axon arborization in both S1 and the S1/S2 border regions. It should be noted that callosal axons with reduced excitability and impaired transmitter release exhibit the overshooting phenotype but not the poor arborization phenotype in the somatosensory cortex. Arrowheads indicate the S1/S2 border. WM, White matter.

or removal of the dorsal thalamus reduces the callosal projections in the S1/S2 border region (Koralek and Killackey, 1990). These findings, together with the results presented here, indicate that both spontaneous and sensory-evoked neuronal activities are critical for the development of callosal projections in the somatosensory cortex.

In addition to a reduction in callosal projections to the border region, our data also demonstrates that Kir2.1 overexpression results in axon overshoot to layer I of both S1 and the S1/S2 border region (Fig. 4), suggesting that correct layer-specific targeting requires spontaneous activity in callosal axons. In contrast, Mizuno et al. (2007) found that the same manipulation in the visual cortex using Kir2.1 overexpression caused only a reduction in callosal projections, mostly as a consequence of the failure of axon arborization in the $17 / 18$ border region. This raises the possibility that neuronal activity has a differential effect on the development of callosal projections in different cortical areas. It remains unclear how neuronal activity affects the development of callosal projections. One possibility is that neuronal activity regulates the expression of membrane receptors for axon guidance cues in the growth cone of these callosal axons, allowing proper axon guidance to their targets. Reduced neuronal activity by Kir2.1 expression may result in a failure of sensing the targetderived "stop" signals, leading to axon overshoot to the superficial layer I. In developing chick spinal cord, blockade or slowing rhythmic bursting of motor neuron activities prevents the forma- tion of normal expression patterns of EphA4 and polysialylated neural cell adhesion molecule on these neurons, and these altered expression patterns lead to motor axon pathfinding errors (Hanson and Landmesser, 2004).

In addition, electrical activity may also regulate neuronal signal transduction pathways, via changes in $\mathrm{Ca}^{2+}$ and cAMP levels, in a manner required for proper growth cone responses of the targetderived guidance cues (Ming et al., 2001). In fact, a recent study shows that spontaneous activity of the retinal ganglionic cells is necessary for the ordering of the retinotopic map in the mouse tectum and cAMP oscillations in growth cones of the ganglion cells are required for the activity blockade-induced suppression of the repellent action of ephrin-A (Nicol et al., 2007). Additional studies will be required to address these possibilities for the guidance of callosal axons. Finally, we noted that the observed effects caused by overexpression of Kir2.1 are likely to be cell autonomous, because untransfected somatosensory callosal projections to Kir2.1electroporated cortex are normal (Y.-Q. Ding, unpublished observations).

\section{Requirement of synaptic activity for callosal axon projection}

Our data further showed that blocking transmitter release from callosal axons also disturbs their projections to the somatosenory cortex, indicating that synaptic activity is also required for the normal development of callosal projections. Interestingly, the effects of neuronal activity and synaptic transmission are not identical and are additive. For example, expression either of Kir2.1 or TeNT-LC reduced callosal projections to the S1/S2 border region, and coexpression led to a more pronounced reduction (Figs. 4, 5, 8), showing an additive function in regulating callosal projection. However, overexpression of Kir2.1 delayed axon midline crossing (Fig. 3), but expression of TeNT-LC had no effect on this process. The delayed midline crossing phenotype found in cotransfected axons resembled that of the Kir2.1 phenotype, indicating that neuronal excitation and transmitter release are differentially involved the callosal axon development. Similarly, the phenotype of axon overshoot and arborization in the superficial layer $\mathrm{I}$ is found in both Kir2.1-expressing axons as well as in cotransfected axons (Figs. 4, 5, 8). Although evoked transmitter release and synaptic transmission depend on the neuronal activity, our results support a hypothesis that neuronal and synaptic activity are involved in regulating different aspects of the development of callosal projections.

Because the reduction in neuronal activity will result in a reduced transmitter release and synaptic activity at the axon terminals, it is possible that, under the present experimental conditions, the reduction in transmitter release caused by Kir2.1 overexpression had not reached the level of reduction caused by TeNT-LC overexpression, leading to some differences between the Kir2.1 and TeNT-LC phenotypes. However, the marked 
withdrawal of TeNT-LC-expressing callosal axons during later developmental stages (Figs. 6, 8) clearly suggests a distinct action of synaptic activity in the stabilization and maintenance of callosal axons in the somatosensory cortex. Synaptic activity may be required for synapse maturation during synaptogenesis (Murphy, 2003; Shen et al., 2006) and plays a stabilizing role during activity-dependent pruning of developing connections (Katz and Shatz, 1996).

In summary, the present study revealed an activity-dependent mechanism underlying the development of callosal projections in the somatosensory cortex. We found that suppression of neuronal activity and blocking transmitter release leads to the disruption of region- and layer-specific callosal projections in the cortex. Our results suggest that neuronal and synaptic activities play additive as well as distinct roles in regulating the development of callosal projections.

\section{References}

Akers RM, Killackey HP (1978) Organization of corticocortical connections in the parietal cortex of the rat. J Comp Neurol 181:513-537.

Brown CE, Dyck RH (2005) Retrograde tracing of the subset of afferent connections in mouse barrel cortex provided by zincergic neurons. J Comp Neurol 486:48-60.

Burrone J, O’Byrne M, Murthy VN (2002) Multiple forms of synaptic plasticity triggered by selective suppression of activity in individual neurons. Nature 420:414-418.

Butler AK, Dantzker JL, Shah RB, Callaway EM (2001) Development of visual cortical axons: layer-specific effects of extrinsic influences and activity blockade. J Comp Neurol 430:321-331.

Cancedda L, Fiumelli H, Chen K, Poo Mm (2007) Excitatory GABA action is essential for morphological maturation of cortical neurons in vivo. J Neurosci 27:5224-5235.

Chen Q, He S, Hu XL, Yu J, Zhou Y, Zheng J, Zhang S, Zhang C, Duan WH, Xiong ZQ (2007) Differential roles of NR2A- and NR2B-containing NMDA receptors in activity-dependent brain-derived neurotrophic factor gene regulation and limbic epileptogenesis. J Neurosci 27:542-552.

Dantzker JL, Callaway EM (1998) The development of local, layer-specific visual cortical axons in the absence of extrinsic influences and intrinsic activity. J Neurosci 18:4145-4154.

Ding YQ, Yin J, Xu HM, Jacquin MF, Chen ZF (2003) Formation of whisker-related principal sensory nucleus-based lemniscal pathway requires a paired homeodomain transcription factor, Drg11. J Neurosci 23:7246-7254.

Ding YQ, Yin J, Kania A, Zhao ZQ, Johnson RL, Chen ZF (2004) Lmxlb controls the differentiation and migration of the superficial dorsal horn neurons of the spinal cord. Development 131:3693-3703.

Hanson MG, Landmesser LT (2004) Normal patterns of spontaneous activity are required for correct motor axon guidance and the expression of specific guidance molecules. Neuron 43:687-701.

Hatanaka Y, Hisanaga S, Heizmann CW, Murakami F (2006) Distinct migratory behavior of early- and late-born neurons derived from the cortical ventricular zone. J Comp Neurol 479:1-14.

Hedin-Pereira C, Lent R, Jhaveri S (1999) Morphogenesis of callosal arbors in the parietal cortex of hamsters. Cereb Cortex 9:50-64.

Hensch TK (2005) Critical period plasticity in local cortical circuits. Nat Rev Neurosci 6:877-888.

Hlushchuk Y, Hari R (2006) Transient suppression of ipsilateral primary somatosensory cortex during tactile finger stimulation. J Neurosci 26:5819-5824.

Hua JY, Smear MC, Baier H, Smith SJ (2005) Regulation of axon growth in vivo by activity-based competition. Nature 434:1022-1026.

Hua SY, Raciborska DA, Trimble WS, Charlton MP (1998) Different VAMP/Synaptobrevin complexes for spontaneous and evoked transmitter release at the crayfish neuromuscular junction. J Neurophysiol 80:3233-3246.
Innocenti GM, Price DJ (2005) Exuberance in the development of cortical networks. Nat Rev Neurosci 6:955-965.

Ivy GO, Killackey HP (1981) The ontogeny of the distribution of callosal projection neurons in the rat parietal cortex. J Comp Neurol 195:367-389.

Ivy GO, Killackey HP (1982) Ontogenetic changes in the projections of neocortical neurons. J Neurosci 2:735-743.

Ivy GO, Could III, Killackey HP (1984) Variability in the distribution of callosal projection neurons in the adult rat parietal cortex. Brain Res 306:53-61.

Johns DC, Marx R, Mains RE, O’Rourke B, Marban E (1999) Inducible genetic suppression of neuronal excitability. J Neurosci 19:1691-1697.

Katz LC, Shatz CJ (1996) Synaptic activity and the construction of cortical circuits. Science 274:1133-1138.

Koralek K, Killackey HP (1990) Callosal projections in rat somatosensory cortex are altered by early removal of afferent input. Proc Natl Acad Sci USA 87:1396-1400.

Kubo Y, Baldwin TJ, Nung Jan Y, Jan LY (1993) Primary structure and functional expression of a mouse inward rectifier potassium channel. Nature 362:127-133.

Li X, Gutierrez DV, Hanson MG, Han J, Mark MD, Chiel H, Hegemann P, Landmesser LT, Herlitze S (2005) Fast noninvasive activation and inhibition of neural and network activity by vertebrate rhodopsin and green algae channelrhodopsin. Proc Natl Acad Sci USA 102:17816-17821.

Ming GL, Henley J, Tessier-Lavigne M, Song HJ, Poo MM (2001) Electrical activity modulates growth cone guidance by diffusible factors. Neuron 29:441-452.

Mizuno H, Hirano T, Tagawa Y (2007) Evidence for activity-dependent cortical wiring: formation of interhemispheric connections in neonatal mouse visual cortex requires projection neuron activity. J Neurosci 27:6760-6770.

Murphy TH (2003) Activity-dependent synapse development: changing the rules. Nat Neurosci 6:9-11.

Nicol X, Voyatzis S, Muzerelle A, Narboux-Neme N, Sudhof TC, Miles R, Gaspar P (2007) cAMP oscillations and retinal activity are permissive for ephrin signaling during the establishment of the retinotopic map. Nat Neurosci 10:340-347.

Olavarria JF, Van Sluyters RC (1995) Comparison of the patterns of callosal connections in lateral parietal cortex of the rat, mouse and hamster. Anat Embryol 191:239-242.

O'Leary DDM, Stanfield BB, Cowan WM (1981) Evidence that the early postnatal restriction of the cells of origin of the callosal projection is due to the elimination of axonal collaterals rather than to the death of neurons. Brain Res 227:607-617.

Paxinos G, Franklin KBJ (2001) The mouse brain in stereotaxic coordinates. New York: Academic.

Ruthazer ES, Akerman CJ, Cline HT (2003) Control of axon branch dynamics by correlated activity in vivo. Science 301:66-70.

Shen W, Wu B, Zhang Z, Dou Y, Rao Zr, Chen YR, Duan S (2006) Activityinduced rapid synaptic maturation mediated by presynaptic Cdc42 signaling. Neuron 50:401-414.

Shuler MG, Krupa DJ, Nicolelis MAL (2001) Bilateral integration of whisker information in the primary somatosensory cortex of rats. J Neurosci 21:5251-5261.

Sur M, Leamey CA (2001) Development and plasticity of cortical area and networks. Nat Rev Neurosci 2:251-262.

Uesaka N, Hirai S, Maruyama T, Ruthazer ES, Yamamoto N (2005) Activity dependence of cortical axon branch formation: a morphological and electrophysiological study using organotypic slice cultures. J Neurosci 25:1-9.

Uesaka N, Ruthazer ES, Yamamoto N (2006) The role of neural activity in cortical axon branching. Neuroscientist 12:102-106.

Wise SP, Jones EG (1976) The organization and postnatal development of the commissural projection of the rat somatic sensory cortex. J Comp Neurol 168:313-344.

Yu CR, Power J, Barnea G, O’Donnell S, Brown HEV, Osborne J, Axel R, Gogos JA (2004) Spontaneous neural activity is required for the establishment and maintenance of the olfactory sensory map. Neuron 42:553566. 